\title{
Discrepancias entre el perfil deseado y alcanzado en el egresado de la carrera de medicina
}

\author{
Gustavo H. Marín
}

Introducción. Las facultades de medicina argentinas acordaron definir un perfil egresado con competencias en atención primaria de la salud (APS). El objetivo del presente estudio es correlacionar el perfil de médico deseado y obtenido.

Materiales y métodos. Estudio descriptivo analítico, con abordaje cualicuantitativo realizado en la carrera de medicina de la Universidad Nacional de La Plata (UNLP), Argentina. Las variables analizadas fueron: motivación, compromiso social, prácticas comunitarias, actividades de extensión, asignaturas cuyo contenido contempla APS (Ciencia Social, Salud Comunitaria, Ecología Humana, Salud Publica), horas de cátedra relacionadas con ellas, cargos de residentes, desempeño en el examen de residencia, cargos de generalistas cubiertos, grado de satisfacción de gestores sanitarios con los egresados, y grado de éxito en prácticas de APS realizada a su población beneficiaria. Análisis estadístico: chi al cuadrado y análisis multivariado.

Resultados. El análisis curricular mostró que sólo el $14,7 \%$ de las asignaturas y el 8,2\% de las horas de la carrera se relacionan con el perfil de APS. Los ingresantes mostraron ser más altruistas que los egresados, quienes fueron más individualistas y carentes del perfil deseado. En sus exámenes de residencia médica, los egresados fallaron en responder preguntas de APS, aunque respondieron correctamente las de otras especialidades. Las vacantes de médicos generalistas quedaron sistemáticamente sin cubrir en el sistema sanitario. La estrategia de APS realizada por egresados a población a cargo fue subóptima (test de Papanicolau: 38,2\%; vacunación: 68,6\%).

Conclusiones. Los egresados de medicina de la UNLP no cumplen con el perfil deseado por la facultad, lo que incide en la futura elección de los puestos de trabajo y en el ejercicio de la profesión.

Palabras clave. Egresado. Estudiante. Médico. Perfil. Residencia.

\section{Gap between the desired and achieved profile in graduates of medical career}

Introduction. All medical school in Argentina agreed in achieve a common profile towards primary health care (PHC) for their graduates. This study was performed in order to determine the correlation between the desired medical profile and the one obtained.

Materials and methods. A descriptive-qualitative and quantitative mixed approach was conducted in the Medical SchoolNational University, La Plata (UNLP), Argentina. Variables studied were: 'motivation for career choice', 'social commitment'; 'community practices', 'PHC practices undertaken by students and graduates', 'amount of courses and teaching hours related with $\mathrm{PHC}^{\prime}$, 'residence posts', 'performance on the residence's exam', 'health managers satisfaction with graduates'. Statistical analysis: data was analyzed by chi-square and multivariate analysis.

Results. Data shows that $14.7 \%$ of the subjects included in the career and $8.2 \%$ of total teaching hours were focuses on PHC profile. Also results indicated that freshmen were more altruistic and had a better profile according to agreed standards than graduates. New doctors showed themselves more self-interested about their own expectations and future. They also chose a medical residency with opposite profile at the one expected, and weren't able to answer basic question related with PHC. Moreover, general practitioners jobs were not taken neither in the residence program nor in the local health care system. Basic PHC practices like PAP-smear or vaccination were insufficiently provided (38.2\% and $68.6 \%$ respectively).

Conclusions. Recent graduates from medical school (UNLP) did not achieve the desired profile. This aspect had a negative impact in the selection of their future jobs and $\mathrm{PHC}$ labor practice performance.

Key words. Graduate. Medical doctor. Profile. Residence. Student.
Facultad de Ciencias Médicas. Universidad Nacional de La Plata. La Plata, Argentina.

Correspondencia:

Dr. Gustavo Horacio Marín. Facultad de Ciencias Médicas. Universidad Nacional de La Plata. Calle 60 y 120 CP 1900. La Plata, Argentina.

E-mail:

gmarin@netverk.com.ar

Agradecimientos:

A la Prof. Lic. Gabriela Marano,

y a las sociólogas Corina Aimetta y Jimena Orchuela, por su apoyo en la elaboración de los instrumentos de medición.

Conflicto de intereses: No declarado.

Competing interests: None declared.

(c) 2014 FEM 


\section{Introducción}

El perfil del egresado es un elemento central en la elección del currículo de una carrera del ciclo universitario [1]. Los aspirantes deben cubrir entonces las expectativas propuestas por las unidades formadoras, las cuales se presupone que a través de un plan de estudios adecuado lograrán generar los suficientes conocimientos, habilidades, actitudes y valores para que sus estudiantes alcancen el perfil de egreso deseado en el campo de la disciplina que corresponda [2]. En este sentido, una serie de debates efectuados en el seno del Ministerio de Educación de la Nación y en el Consejo Universitario se llevaron a cabo con el fin de elaborar y definir estándares para las carreras de medicina, que culminaron con la sanción de la Resolución MCE n. ${ }^{\circ}$ $535 / 99$, donde quedaron establecidos para la carrera de medicina los contenidos curriculares básicos, los criterios sobre intensidad de la formación práctica, el alcance del título de médico y sus respectivos estándares de acreditación, en los términos del artículo 43 de la Ley de Educación Superior, reconociéndose la atención primaria de la salud (APS) como base para cumplir con los mismos [3]. Estos contenidos y cargas horarias mínimas se confirmaron años después a través de la Resolución MECyT 1314/07. También el Ministerio de Salud de la Nación definió mediante su Plan Federal de Salud el mismo perfil para la formación de recursos humanos en salud, expresando que 'la atención primaria será la prioridad absoluta y las acciones de promoción y prevención obtendrán una asignación creciente de recursos. Promover la formación de profesionales con énfasis en medicina generalista, o su reconversión, a fin de contar con equipos de salud con capacidad de intervención en las prioridades definidas por las políticas sanitarias de este plan' [4]. En consonancia, la Facultad de Ciencias Médicas de la Universidad Nacional de La Plata (UNLP) se adhirió a dicho perfil planteando formar 'un médico general, definido como el profesional que está capacitado en conocimientos, habilidades y actitudes para la protección de la salud en niños y adultos y para la asistencia, recuperación y rehabilitación en adultos, remarcando la importancia de la APS como estrategia longitudinal en toda la carrera' [5]. Estos aspectos fueron tiempo después refrendados no sólo por la Facultad de Medicina de la UNLP, sino por todas las demás facultades de medicina pública de Argentina, reafirmando un perfil de generalista con orientación en la APS para sus egresados [6]. La Comisión Nacional de Evaluación y Acreditación Universitaria (CONEAU), adhirién- dose a esta idea, audita periódicamente el cumplimiento de los estándares básicos para las carreras de medicina con el fin de garantizar que ese perfil se vea reflejado en los currículos de toda carrera de salud [7]. La Organización Mundial de la Salud también promueve la formación de un profesional generalista que base sus acciones en la promoción de la salud, la prevención de enfermedades y la atención de patologías prevalentes en la comunidad, es decir, ni más ni menos que en la APS [8]. Sin embargo, no existe aún una evaluación de los egresados que permita confirmar si efectivamente ese perfil que figura en las intenciones, se plasma en la realidad [9]. Por otro lado, las instituciones que conforman el Sistema de Atención de la Salud requieren perfiles similares a los que las unidades académicas dicen formar [10]; no obstante, con frecuencia, la oferta de puestos en dichos sectores suele no cubrirse en su totalidad con los recientemente egresados.

Con el objetivo de evaluar en qué medida el perfil del egresado se correlaciona con el perfil de profesional deseado por la institución formadora ( $\mathrm{Fa}$ cultad de Ciencias Médicas de la UNLP) y cuál es el impacto que tiene este perfil en la elección de la formación de posgrado y en la cobertura de puestos de trabajo solicitados por el sistema sanitario, se inició el siguiente estudio que se detalla a continuación.

\section{Materiales y métodos}

\section{Tipo de estudio}

Se trata de un estudio transversal del tipo descriptivo analítico, con abordaje cualicuantitativo (mixedCreswell) [11].

\section{Objeto de estudio}

Perfil de los ingresantes y egresados de la carrera de medicina de la UNLP pertenecientes al plan de estudios conocido como 'plan viejo' (vigente desde 1992), desempeño de los egresados en el examen de residencias médicas, y demanda de puestos de trabajo para generalistas tanto en residencias como en el sistema de salud.

\section{Lugar y período de estudio}

Facultad de Ciencias Médicas de la UNLP, Argentina, del 1 de enero de 2007 al 31 de diciembre de 2010 . 


\section{Variables}

Las variables seleccionadas se relacionan con parámetros de ingreso (input), proceso, resultado (output) e impacto (outcome):

- Variables de input o ingreso: motivación de estudiantes para estudiar medicina, compromiso social de los ingresantes, cátedras en relación directa con la formación en APS, horas de cátedra destinadas a la formación del perfil generalista/ APS, cargos de residentes ofrecidos para medicina general, cargos ofrecidos para generalistas en el sistema local de salud.

- Variables de proceso: prácticas comunitarias de APS realizadas por los estudiantes, actividades de extensión comunitaria realizadas por los alumnos durante la carrera.

- Variables de output o resultados: motivación de los egresados para ejercer una medicina relacionada con la APS, compromiso social de los egresados, cantidad de cupos de generalistas ocupados en la residencia médica, cantidad de preguntas correctamente respondidas en relación con la formación de generalista/APS en el examen de residencia, cargos de generalistas cubiertos en el sistema de salud local.

- Variables que objetivan el outcome o impacto: grado de satisfacción de las autoridades sanitarias con el desempeño de los nuevos médicos, realización de prácticas de APS por parte de los egresados al incorporarse a un puesto de trabajo tomándose como ejemplos el test de Papanicolau (PAP) realizado en mujeres mayores de 30 años y la vacunación (cumplimiento del cronograma oficial) en niños menores de 14 años de la población a cargo (periodo 2007-2010).

Un resumen de las variables y su forma de medición se puede apreciar en la tabla I.

\section{Instrumentos para recogida de datos}

- Entrevistas a ingresantes y egresados (seleccionados aleatoriamente).

- Encuesta a ingresantes.

- Encuesta a egresados: ambas encuestas constaron de 20 preguntas, fueron autoadministradas, en formato papel y se realizaron a ingresantes y graduados seleccionados de forma aleatoria durante el período 2007-2010. Las preguntas de ambos instrumentos se relacionaban con los motivos que los llevaron a elegir la carrera (vocación social, impronta familiar, aspectos económicos), expectativas respecto a su futuro ejercicio profe- sional (lugar deseado para ejercer su profesión, tipo de especialidad a elegir, grado de ponderación del reconocimiento comunitario, estatus social, bienestar económico) y autopercepción respecto al papel que debe cumplir el médico en la comunidad (aspectos sobre compromiso comunitario, relación del entrevistado/encuestado con actividades comunitarias y medicina social). Las respuestas se tabularon según sexo, y tipo de respuesta (datos disponibles en http://www.med. unlp.edu.ar//introduccion.php?itemc=Introduc ción\&idc=7\&idl=348\&idbot=3).

- Programa de la carrera de medicina de la UNLP determinando número de asignaturas y horas destinadas a la medicina general, familiar y social.

- Planilla de registro de desempeño en el examen de admisión a las residencias médicas en la provincia de Buenos Aires, que consta de 70 preguntas de opción múltiple, de las cuales se tomaron de forma aleatoria estratificada 10 de ellas consideradas de dificultad moderada por el departamento de pedagogía (cinco destinadas a explorar conocimientos de APS y cinco sobre conocimientos puntuales de las especialidades). Se tomó como modelo el examen de residencia del año 2008 (disponible en http://residenciasmedicas.com.ar/provincia/examen-provincia-de-bsas-ano-2008).

- Registro de puestos ofrecidos y de puestos cubiertos en cargos de residentes (base de datos de la Dirección de Capacitación de Profesionales de la Salud del Ministerio de Salud de la provincia de Buenos Aires en los años 2007-2010).

- Registro municipal de puestos de médicos para APS (ofrecidos y efectivamente contratados en el período 2007-2011).

- Entrevista a autoridades sanitarias. Se entrevistó a las máximas autoridades de Salud municipal y referentes de regiones sanitarias respecto a su visión sobre la problemática de los recursos humanos en el primer nivel de atención de la salud.

La categorización de las herramientas de medición se puede observar en la tabla II.

\section{Tamaño de la muestra}

Se determinó el número requerido para realizar encuestas a ingresantes y egresados mediante la siguiente fórmula:

$n=N 3 Z 23 p(1-p) / d 23(N-1)+Z 23 p(1-p)$,

donde $n$ es el tamaño de la muestra; $N$, la población 
Tabla I. Tipo de variables y forma de medición de cada una.

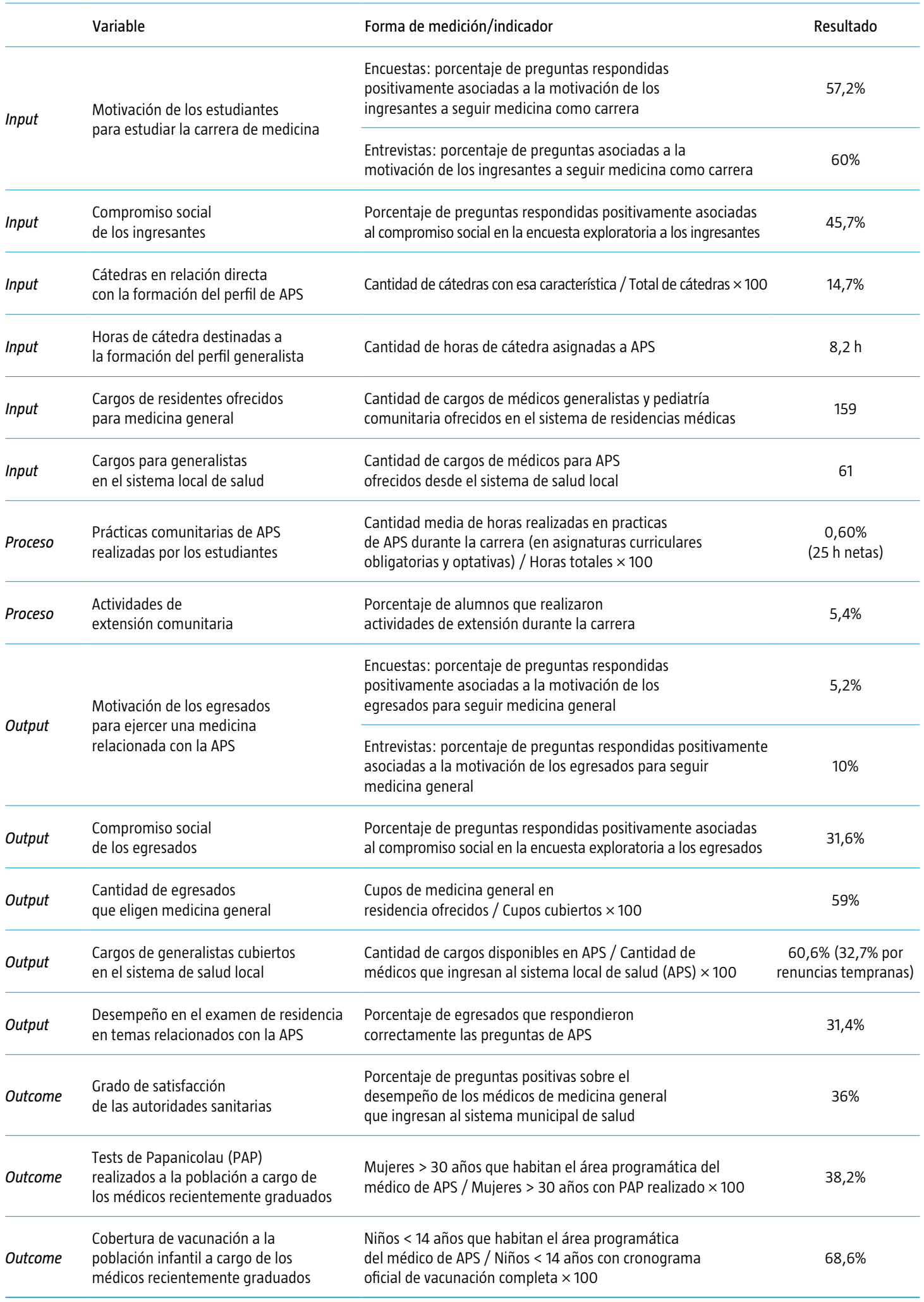


total de alumnos de medicina; $Z$, el valor de $z$ para el nivel de confianza $(1-\alpha)$; $p$, la proporción esperada para las variables elegidas, y $d$, la precisión absoluta.

El número mínimo requerido de encuestas teniendo en cuenta la fórmula de muestreo mencionada fue de 120 (aunque finalmente en el trabajo se triplicó dicho valor).

\section{Análisis estadístico}

Los resultados de las encuestas se expresan en medias para las variables continuas y en porcentajes para variables categóricas. La comparación entre las respuestas brindadas por ingresantes y egresados se analizó con $\chi^{2}$. Se realizó un análisis multivariado para determinar las diferencias obtenidas entre ingresantes y egresados.

\section{Resultados}

En la Facultad de Ciencias Médicas de la UNLP ingresan anualmente en la carrera de medicina 300400 alumnos tras un proceso de selección. El análisis curricular del plan de 1992 de dicha carrera mostró que un 8,2\% de las horas de cátedra (342 h de las 4.124 h totales) y un $14,7 \%$ de las 34 asignaturas se relacionan con el perfil de APS buscado para los futuros egresados, considerando entre ellas: Ciencias Sociales; Salud, Medicina y Sociedad I y II; Higiene, Medicina Preventiva y Social I y II. Buscando ponderar dicho perfil, el Ministerio de Educación, mediante la Resolución n. ${ }^{\circ}$ 535/99, efectuó recomendaciones para introducir modificaciones al plan de estudios de la carrera. Siguiendo dichas consignas, se cambió en el año 2004 el plan de estudios ('plan nuevo'), que tiene 52 materias, de las cuales siete son electivas. Todas las asignaturas del nuevo plan constituyen $4.670 \mathrm{~h}$, de las cuales $460 \mathrm{~h}(9,8 \%)$ se relacionan con el perfil buscado. No obstante, se incorporaron $1.600 \mathrm{~h}$ en concepto de práctica final obligatoria, en la cual el alumno debe realizar prácticas en hospitales (en las cuatro especialidades básicas de clínica, pediatría, ginecología y cirugía) y 320 h en los centros de APS. El impacto de este cambio no podrá evaluarse en este documento, ya que esta práctica final obligatoria se encuentra al final de la carrera y los alumnos del nuevo plan actualmente están llegando a esa instancia.

La evaluación del perfil de ingresantes se realizó a través de 10 entrevistas y 506 encuestas a nuevos alumnos. Los resultados obtenidos a través de las
Tabla II. Resumen de las herramientas utilizadas para la recogida de datos: datos primarios.

\begin{tabular}{|c|c|}
\hline \multirow{3}{*}{$\begin{array}{l}\text { Metodología } \\
\text { cuantitativa }\end{array}$} & Encuestas estructuradas a ingresantes y egresados \\
\hline & Planilla de registro de desempeño del examen de residencia \\
\hline & Registro de puestos de generalista ofrecidos y ocupados en municipio \\
\hline \multirow{3}{*}{$\begin{array}{l}\text { Metodología } \\
\text { cualitativa }\end{array}$} & Entrevistas a ingresantes (sobre sus expectativas de la profesión) \\
\hline & $\begin{array}{l}\text { Entrevistas a egresados (sobre sus expectativas de la profesión, } \\
\text { elección, satisfacción y percepción de capacidades adquiridas) }\end{array}$ \\
\hline & Entrevistas a autoridades sanitarias (sobre el perfil de médico que reciben en el sistema) \\
\hline
\end{tabular}

encuestas mostraron que un $58,3 \%$ de los ingresantes deseaba volver a ejercer la medicina en su pueblo o barrio de origen y ser allí un médico generalista. Asimismo, las encuestas mostraron que el $57,2 \%$ de los ingresantes inició la carrera por motivos altruistas como 'ayudar al prójimo', 'garantizar el derecho a la salud de la comunidad' o 'solucionar los problemas a la gente' (Tabla III). De las entrevistas a ingresantes surgió que el 60\% de ellos eligió la carrera para ayudar a otros y ese mismo porcentaje esperaba elegir como especialización medicina social, familiar o general, un aspecto que también se objetivó en las encuestas (Tabla III).

El perfil de los egresados se evaluó mediante 349 encuestas a médicos en el momento de su graduación. Se objetivó que sólo el 5,4\% de los egresados realizó durante su carrera actividades comunitarias como voluntariado o proyectos de extensión o actividades en organizaciones no gubernamentales. El $57,9 \%$ de los egresados admitió que asocia la elección de la carrera de medicina a motivos de crecimiento individual y personal, objetivados en frases como 'para seguir la tradición familiar', 'porque ser médico da prestigio social' o porque la profesión permitirá 'tener una mejor remuneración económica' (Tabla III). Al momento de elegir una rama de la medicina, los egresados se manifestaron mayormente a favor de las especialidades y en menor medida se volcaron a las ramas asociadas a la APS (Tabla IV). Las entrevistas realizadas a egresados mostraron que en un 70\% primaban los aspectos económicos al elegir una especialidad para ejercer la profesión. A través del análisis multivariado se estableció que las respuestas brindadas en las encuestas a ingresantes y egresados no tuvieron diferencias estadísticas significativas en relación al sexo, origen, tipo de formación media (publica o privada) y convivencia de los encuestados (con familiares, amis- 
Tabla III. Motivos de la elección de la carrera de medicina.

\begin{tabular}{|c|c|c|c|c|}
\hline & Ingresantes & Egresados & $p$ & Perfil \\
\hline Para garantizar el derecho a la salud de otros & $9,8 \%$ & $5,7 \%$ & 0,04 & \\
\hline Para ayudar al prójimo & $27,9 \%$ & $17,8 \%$ & 0,01 & Altruista \\
\hline Para solucionar los problemas de la gente & $19,5 \%$ & $11,6 \%$ & 0,02 & \\
\hline Por remuneración y ascenso social & $9,6 \%$ & $15,8 \%$ & 0,02 & \\
\hline Por prestigio & $12,5 \%$ & $22,1 \%$ & 0,01 & Individualista \\
\hline Por tradición familiar & $17,2 \%$ & $20,8 \%$ & NS & \\
\hline
\end{tabular}

NS: no significativo.

Tabla IV. Rama o especialidad preferida como intención para ejercer la profesión.

\begin{tabular}{lccc}
\hline & Ingresantes & Egresados & $p$ \\
\hline Anestesiología & $0,8 \%$ & $19,4 \%$ & $<0,0001$ \\
\hline Cirugía-ginecología & $6,4 \%$ & $17,8 \%$ & $\mathrm{NS}$ \\
\hline Clínica médica & $18,1 \%$ & $11,0 \%$ & $<0,0001$ \\
\hline Medicina familiar & $27,2 \%$ & $2,1 \%$ & $<0,0001$ \\
\hline Medicina general & $21,3 \%$ & $2,7 \%$ & 0,04 \\
\hline Medicina social/sanitario & $8,7 \%$ & $0,2 \%$ & $<0,0001$ \\
\hline Neuro/Cardio/Neumología & $3,1 \%$ & $30,9 \%$ & $\mathrm{NS}$ \\
\hline Neurocirugía & $1,1 \%$ & $4,8 \%$ & $\mathrm{NS}$ \\
\hline Trasplante & $1,3 \%$ & $3,4 \%$ &
\end{tabular}

NS: no significativo. alguna especialidad o a una situación menos prevalente. Utilizando una planilla de registro destinada a evaluar el desempeño en las preguntas efectuadas, se exploraron las respuestas del examen de residencia. De su análisis surge que los postulantes desconocían en su mayoría $(68,6 \%)$ las preguntas básicas relacionadas con la promoción de la salud y la prevención primaria y secundaria de las enfermedades (estrategia de APS), mientras que el 69,71\% de los postulantes contestó correctamente preguntas puntuales en relación con conocimientos específicos sobre patologías muy poco prevalentes en nuestra comunidad (Tabla V).

Por otra parte, el análisis de los cargos de residentes ofrecidos y cubiertos mostró que en medicina general, al finalizar la adjudicación, siempre existen puestos vacantes sin cubrir (34-40\%), mientras que en especialidades muy específicas como anestesia o dermatología existe una demanda seis veces superior a la oferta (más de 200 postulantes para sólo 36 cargos). Con respecto al mercado laboral se observó que los puestos solicitados por el sistema de salud local son en su mayoría para médicos generalistas. Existen 1.870 centros de APS que necesitan al menos cuatro médicos cada uno de ellos. Es decir, hay una demanda teórica de 7.480 médicos con dicho perfil para desempeñarse en el primer nivel de atención de Buenos Aires. Sin embargo, nuestro equipo de trabajo ha demostrado que sólo un 32\% de los centros de APS provinciales poseen un médico generalista [12]. Para objetivar la oferta de estos puestos de trabajo en APS y la cobertura de los mismos en el ámbito regional, se revelaron a modo de ejemplo los cargos ofrecidos por el primer nivel de atención en la capital de la provincia de Buenos Aires (municipio de La Plata). Existen allí 44 centros de APS y se llamó en el período de estudio a 61 cargos de médicos generalistas o pediatras. Sólo se lograron cubrir 37 de los 61 puestos en los últimos cinco años, y a poco de ingresar, 17 de los nuevos profesionales renunciaron (14 de ellos para cubrir un puesto específico en un hospital de alta complejidad fuera de su perfil de generalista, y tres optaron por una segunda residencia para especializarse en otra rama de la medicina). En las entrevistas realizadas a las autoridades sanitarias se exponen algunas interpretaciones para esta situación: 'la verdad es que uno piensa que un joven profesional, a quien se le asigna un lugar de trabajo en un barrio en el cual sus conocimientos pueden ser sumamente útiles para la comunidad, estaría más que conforme de ejercer allí [...]; sin embargo, parecen no querer realizar esa labor o bien no tener experiencia para desempeñarla'; 'es cierto 
Tabla V. Análisis de las respuestas brindadas por los postulantes en el examen de residencia.

\begin{tabular}{|c|c|c|c|}
\hline $\begin{array}{l}\text { Tipo de } \\
\text { pregunta }\end{array}$ & Pregunta & $\begin{array}{l}\text { Respuestas } \\
\text { correctas }\end{array}$ & $\begin{array}{l}\text { Media según tipo } \\
\text { de pregunta }\end{array}$ \\
\hline $\mathrm{E}$ & El sistema de clasificación sexual de Tanner... & $70,40 \%$ & \multirow{5}{*}{$69,71 \pm 4,86 \%$} \\
\hline $\mathrm{E}$ & $\begin{array}{l}\text { En el carcinoma de vulva, ¿cuál es el factor independiente de metástasis } \\
\text { ganglionares más importante según los criterios histológicos del GOG? }\end{array}$ & $62,20 \%$ & \\
\hline $\mathrm{E}$ & En relación a la cefalea primaria tensional, indique la opción correcta... & $77,30 \%$ & \\
\hline $\mathrm{E}$ & La localización más frecuente del cáncer de vulva es... & $68,10 \%$ & \\
\hline $\mathrm{E}$ & $\begin{array}{l}\text { La monitorización cardiaca con holter ha tenido últimamente considerable desarrollo en } \\
\text { sus aplicaciones clínicas. Indique de las siguientes cuál no correspondería con una de ellas }\end{array}$ & $71,11 \%$ & \\
\hline B & $\begin{array}{l}\text { La mortalidad perinatal abarca un período que } \\
\text { comienza a las } 20 \text { semanas de gestación y termina a las... }\end{array}$ & $32,75 \%$ & \multirow{5}{*}{$31,40 \pm 7,06 \%$} \\
\hline B & La categoría de glucemia alterada en ayunas se define por una de las siguientes cifras... & $40,26 \%$ & \\
\hline B & La vacuna BCG se administra dentro de la primera semana de vida y... & $33,45 \%$ & \\
\hline B & $\begin{array}{l}\text { ¿Cuáles de las siguientes son las mayores causas } \\
\text { de la mortalidad materna e infantil en nuestro país? }\end{array}$ & $29,74 \%$ & \\
\hline B & $\begin{array}{l}\text { Usted es el responsable de salud de una comunidad y conoce a la población de } \\
\text { su área programática. Llega el otoño y la bronquiolitis aguda. ¿Cuál de los siguientes } \\
\text { grupos de niños debería considerarse de riesgo de padecer bronquiolitis? }\end{array}$ & $20,82 \%$ & \\
\hline
\end{tabular}

E: preguntas dirigidas a un conocimiento puntual y a contenidos de una especialidad médica distinta a la de medicina general y familiar; B: preguntas básicas dirigidas a evaluar el conocimiento de un médico generalista.

que la remuneración que se le brinda a los profesionales en los municipios es menor que la que pueden ganar en un hospital provincial'; 'también es cierto que la población está muy agresiva y que reacciona mal ante algunas situaciones y son los profesionales de la salud los destinatarios de esas agresiones [...]. Basta como ejemplo lo que sucedió en el CIC (Centro de Integración Comunal ubicado en Los Hornos). La jefa del centro pretendió dar participación en las decisiones sanitarias a la propia comunidad organizando la mesa de barrio. Grupos locales enfrentados entre sí, participantes en esa mesa, finalmente terminaron por agredirla verbal y físicamente, lo cual provocó su renuncia y la aceptación de un puesto hospitalario'; 'es como que en el hospital se sienten más protegidos, más acompañados, la relación con la gente es mas indirecta.'

La tasa de PAP realizados en mujeres mayores de 30 años que habitaban en las áreas programáticas a cargo de los jóvenes egresados que se desempeñaron en un centro de APS de La Plata fue del $38,2 \%$. Es decir, la mayor parte de la población de sexo femenino a cargo de estos nuevos profesionales no tenían realizado dicho control básico de APS al cabo del año. Por otro lado, la cobertura de vacunación de la población de niños menores de 14 años a cargo de los mismos profesionales fue del 68,6\% para una cobertura media esperada para el municipio del $98,7 \%$ (Tabla I).

\section{Discusión}

La planificación basada en las competencias sostenida en los planes de estudio tiene un rol fundamental en la educación superior. Sin embargo, existe un currículo oculto de los egresados que no siempre responde al perfil esperado por las unidades académicas o el mercado laboral. Este trabajo justamente expone una situación en la cual el perfil de graduado deseado aún no se corresponde al real, aspecto que se refleja luego en la formación de posgrado y en la práctica profesional. En concordancia con el perfil que exige el sistema sanitario, las uni- 
versidades nacionales han coincidido en que el perfil de médico que desean obtener es uno relacionado con la APS. A partir de esta decisión, en la última década se incorporaron a los planes de estudio materias de base humanística, social o que abordan la estrategia de APS en asignaturas como 'Sociales, Salud y Medicina Comunitaria, Salud Ambiental, Ecología Humana, Salud Pública I y II. El incremento de asignaturas con temáticas sociales realizado en la UNLP (plan de 2004) paradójicamente redujo la carga horaria de asignaturas relacionadas con la APS, aunque incorporó la práctica final obligatoria, que abre una esperanza para poder cambiar el escenario anterior. No obstante, los planes de estudio no aseguran por sí mismos los resultados esperados. Al respecto cabría reflexionar sobre las causas por las cuales un alumno, tras permanecer siete años en una universidad, no logra incorporar el mensaje emanado de la unidad académica en la cual estudió. Se debe considerar como posibles respuestas que los docentes no están preparados para formar ese perfil de profesional que requiere la sociedad, o bien que los alumnos tienen en sus docentes modelos inadecuados para reflejarse. Respecto a este punto, la actividad médica ha ido acompañando los cambios sociales. La otrora satisfacción del galeno en velar por la salud de su comunidad, en observar el resultado benéfico de sus saberes en sus pacientes, y el sentirse bien pagado sólo al ver estos logros, ha cambiado indudablemente en nuestros días. Aquel maestro/docente/médico que para su comunidad era un referente ético y solidario, y que por ello constituía un ejemplo de vida y modelo de profesional exitoso para los jóvenes estudiantes, ha dado paso en la actualidad a otros modelos de éxito [13]. El profesional con alto poder adquisitivo, con acceso a bienes materiales y a tecnologías de alta complejidad, es hoy un modelo en el cual muchos alumnos pretenden convertirse al momento de graduarse. El compromiso, la entrega y la visión colectiva han quedado desplazados por la rentabilidad, el individualismo y el éxito económico. No es raro entonces que los egresados opten por especialidades asociadas a altos ingresos económicos, como anestesiología, cirugía estética o dermatología, para formarse en sus posgrados.

Por lo expuesto, resulta necesario articular los espacios de formación de posgrado, como las residencias [11], con las instituciones formadoras de grado y con los servicios de salud que aceptan dichos recursos humanos [13]. Asimismo resultará fundamental que las instituciones formadoras en salud tengan en cuenta las necesidades del sistema sanitario para definir el perfil de sus egresados, re- formular sus planes de estudio y seleccionar las estrategias de enseñanza.

El sistema de salud argentino requiere médicos capaces de brindar una atención integral y eventualmente derivar al especialista si la situación lo requiere. Es esta una de las razones por las cuales las facultades de medicina de las universidades nacionales de Argentina han coincidido en que sus graduados deben tener un perfil de médico generalista con capacidades de aplicar la estrategia de APS. Sin embargo, a la luz de los resultados del presente estudio, la formación de nuevos médicos va a contramano de estas necesidades sanitarias.

Entre las posibles explicaciones de esta realidad no se puede dejar de analizar la inequidad en la remuneración económica que reciben los médicos de las distintas especialidades. La capacidad de pago de los municipios (encargados de la estrategia de APS) es históricamente muy limitada y ello influye negativamente en la convocatoria a nuevos profesionales para cubrir cargos de generalistas. Parece paradójico que los efectores públicos necesiten ese perfil pero luego ofrezcan un salario magro por él: 287 euros al mes por 35 h de trabajo semanales (valores de marzo de 2012), mientras que un ingresante hospitalario de otras especialidades tienen un sueldo inicial que duplica esa cantidad (612 euros).

La conjunción de la falta de preparación para ejercer el rol profesional en el primer nivel de atención, la escasa remuneración de estos profesionales y la relación directa que tienen los médicos con los problemas sociales de la comunidad, forman una compleja relación que mina el estímulo que puedan tener los nuevos profesionales en internalizar ese nuevo perfil y termina por ubicar a los egresados en zonas urbanas donde hay ya gran densidad de profesionales, buscando su propio 'nicho' al elegir el ejercicio de una subespecialidad de la medicina.

Teniendo en cuenta que en el año 2004 la facultad decidió seguir las recomendaciones del Ministerio de Educación incorporando al plan de estudios la práctica final obligatoria, que posee un $50 \%$ de actividades directamente relacionadas con la APS, es posible que ésta pueda cumplir un papel fundamental en el futuro, ya que los jóvenes estudiantes, al ser asignados a distintas localidades, tendrán un contacto con la medicina general y familiar y un mayor vinculo afectivo con la comunidad. Este hecho podrá modificar los perfiles y estimular a los egresados a radicarse definitivamente en esos lugares. Será entonces interesante repetir esta evaluación en el futuro, una vez que los graduados del plan nuevo hayan cumplido con dicha actividad. 
Bibliografía

1. Díaz-Barriga A. Currículum. Tensiones conceptuales. Rev Elect Inv Educ 2003; 2: 21-7.

2. De Alba A. Currículum-sociedad. Instituto de Investigaciones sobre la Universidad y la Educación. México DF: Plaza y Valdés; 2007.

3. Boletín Oficial de la Republica Argentina. Resolución 535/99 del Ministerio de Educación. URL: http://www.boletinoficial. gov.ar/DisplayPdf.aspx?s=01\&f=19990825.

4. Ojea O, Barragán H. El plan federal de salud. Fundamentos de Salud Pública 2008; 2: 411-7.

5. Fundamentos de la reforma curricular en la Facultad de Ciencias Médicas de la UNLP. URL: http://www.med.unlp. edu.ar//archivos/fundamentos_y_contenidos_de_la reforma_curricular.pdf.

6. Ministerio de Salud. Artículo de prensa. 'Promueven formar médicos generalistas con orientación en salud primaria'. Foro Argentino de Facultades y Escuelas de Medicina Pública. URL: http://www.prensa.argentina.ar/2012/06/24/31798promueven-formar-medicos-generalistas-con-orientacionen-salud-primaria.php.

7. Organización Panamericana de la Salud-Organización Mundial de la Salud. La acreditación de programas de formación en medicina y orientación hacia APS. Renovación de la Atención Primaria de la Salud en las Américas 2010; 3: 41-59.

8. Martínez-Marull A, Fernández CR, Jeppesen C, Caillon A Los procesos de acreditación de carreras de medicina en la República Argentina. Análisis crítico de los estándares, de los procedimientos y de los resultados de la evaluación. Buenos Aires: CONEAU; 2000. URL: www.coneau.edu.ar/ archivos/1229.pdf.

9. Perfil solicitado en el sistema de residencias públicas de la provincia de Buenos Aires. Ministerio de Salud de Buenos Aires; 2008. URL: www.ms.gba.gov.ar/servicios/Residencias Basicas2008.

10. Vallese MC, Roa R. Primer censo de residencias de medicina familiar y general en la República Argentina. Arch Med Fam General 2005; 1: 25-38.

11. Creswell J. Research design. Qualitative, quantitative, and mixed methods approaches. 4 ed. Los Ángeles: Sage; 2013.

12. Marin GH, Silberman M, Sanguinetti C. The quality of primary care health centres in Buenos Aires, Argentina. Qual Prim Care 2009; 17: 283-7.

13. De Espínola H, Melis BI. Los graduados de medicina evalúan la pertinencia de la formación médica. Facultad de Medicina-UNNE. URL: http://www.unne.edu.ar/Web/cyt/ cyt/2002/01-Sociales/S-054.pdf.

14. González-García G. Médicos: la salud de una profesión. Buenos Aires: Salud; 2012. 